\title{
FLORISTIC DIVERSITY OF THE “ЕĄKI W KOMBORNI” NATURA 2000 SITE PLH180042 (WESTERN CARPATHIANS)
}

\author{
MARia Ziaja, TOMASZ WÓJCIK
}

\begin{abstract}
M. Ziaja, T. Wójcik, Department of Natural Sciences, University of Rzeszów, Cicha 2 A, 35-326 Rzeszów, Poland, e-mail: mziaja@ur.edu.pl
\end{abstract}

(Received: February 27, 2017. Accepted: April 10, 2017)

\begin{abstract}
Aвstract. The vascular flora in the "Łąki w Komborni" Natura 2000 site comprises 219 species representing 44 families and 147 genera. Species from the families Asteraceae, Poaceae, Lamiaceae, Fabaceae, Rosaceae, Polygonaceae, Cyperaceae, Apiaceae, and Ranunculaceae were found to dominate. A geographical and historical analysis of species confirmed the clear dominance of apophytes (84\%) over anthropophytes (16\%) as well as perennials $(69 \%)$ over short-lived $(23 \%)$ species. The biological spectrum was dominated by hemicryptophytes (54\%) and therophytes (19\%), compared with other life forms. In terms of synecology, the flora was mainly represented by species from the classes Molinio-Arrhenatheretea, Stellarietea mediae, Artemisietea vulgaris and Phragmitetea. The "Łąki w Komborni" site is a habitat for 82 (37\%) medicinal species and $155(70 \%)$ forage species for bees. Noteworthy is the occurrence of protected species, e.g. Gladiolus imbricatus, Trollius europaeus, Centhaurium erythraea, Dactylorhiza majalis, Platanthera bifolia and Primula elatior.
\end{abstract}

KeY WORDS: Natura 2000 site, meadows, floristic diversity, vascular plants, south-eastern part of Poland

\section{INTRODUCTION}

Meadows are semi-natural communities associated with human activity; they play an important role in the conservation of biodiversity and in the agricultural landscape (WolańsKi \& TrąBA 2007, KĄCKI 2012, Trąa \& Wolański 2012, ZarZYCKI \& KorZeniaK 2013, KotAŃSKA et al. 2015).

Meadow communities have a complex structure and a variable dynamics of growth and development. The high nature value of meadow ecosystems is related to their phytocoenotic diversity and species richness, in particular the presence of many rare and protected species (BABCZYŃSKA-SENDEK 2009, NowAK et al. 2015, WójciK \& JANicKa 2016, Ziaja \& WóJCiK $2016 \mathrm{a}, \mathrm{b})$. The floristic diversity of meadows depends on natural habitat factors and human activity (regulation of water relations, fertilisation, treatments, mowing and sward grazing) (ElLenberg 1988, KotaŃSKa 1993, KrysZAK \& KRYSZAK 2007, SPYCHALSKI et al. 2011). Among semi-natural habitats, meadows are indicated as valuable biotopes for pollinator restoration (Wrzesień \& Denisow 2006a, Denisow \& WRZESIEŃ 2007).
In the recent decades, meadows have undergone rapid transformations both in Poland and in Europe. Disappearance of meadow communities and loss of biodiversity have been reported (BATOR 2005, PosCHLOD et al. 2005, ZAEUSKI 2007, LiNDBORG et al. 2008, Trąa \& Wolański 2012, EuRopean Red List... 2016); they were mainly caused by anthropogenic factors, e.g. abandonment or intensification of land use and changes in the water relations (KoTAŃSKA 1993, ZAŁUSKI 2011, KĄCKI 2012). These processes result in changes in the meadow species composition, initiation of secondary succession, an increase in the share of expansive species and reduction of species diversity (MichalsKa-Hejduk \& Kopeć 2012, Kulik 2014).

The semi-natural ecosystems of Molinia meadows are most important for preservation of biodiversity and most endangered in Europe (KącKi \& MichalSKa-Hejduk 2010, Nowak et al. 2015). These meadows are regarded as one of the species-richest ecosystems; they are characterised by the highest nature values and offer a habitat for many rare plant species (Kącki \& Michalska-Hejduk 2010, Ziaja \& Wójcik 2016a, b). Given the high natural values and the high degree of threat in Europe, wet meadows have been included in the list of habitats protected under the 
Natura 2000 network as habitat type 6410 (Council Directive $92 / 43 /$ EEC on the conservation of natural habitats and wild fauna and flora).

The aim of the study was to know flora richness and diversity in the protected area.

\section{MATERIAL AND METHODS}

The field study was conducted in the "Łąki w Komborni” Natura 2000 site PLH180042 (49²41'N, $21^{\circ} 51^{\prime} \mathrm{E}$ ) in 2015-2016 (Fig. 1). The study area is situated in Iskrzynia, Krościenko Wyżne Commune, Krosno County, Podkarpackie Province. According to the physical-geographical division of Poland (KoNDRACKI 2012), the study area is part of the Jasielsko-Krośnieńska Basin mesoregion, Środkowobeskidzkie Foothills macroregion in the Outer Western Carpathians. The aim of protection of the Natura 2000 site is to preserve intermittently wet meadows from the alliance Molinion caeruleae and three species of butterflies from the Annex II of the Habitats Directive: Lycaena dispar, Maculinea teleius, $M$. nausithous. The entire complex covers an area of 13.14 ha. Habitat 6410 occupies over $50 \%$ of the area (7.17 ha) and the conservation status of meadows has been evaluated as unsatisfactory (U1) (PZO Łąki w Komborni PLH 180042). The vascular plant nomenclature followed that proposed by Mirek et al. (2002), the names of the families were adopted from RUtKowski (2004), and the phytosociological classification was based on that developed by MatuszKIEwICZ (2005). The geographical-historical status was specified in accordance with ZAJAc (1979) and ToKARSKA-GUZIK (2005). The classification of life forms (according to Raunkiaer) was provided as in ZARZYCKI et al. (2002). Classification into medicinal and forage plant groups followed LiPIŃsKI (1958), RUTKOWSKI (2004) and Wrzesień \& Denisow (2006b).

\section{RESULTS AND DISCUSSION}

The vascular plant flora in the study area comprised 219 species representing 44 families and 147 genera (Table 1). The most numerous were representatives of the families Asteraceae (30 species), Poaceae (26), Lamiaceae (14), Fabaceae (12), Rosaceae (12), and Polygonaceae (10), which accounted for $47 \%$ species in total (Table 2). A large number of families were represented by two or three species and 13 families by a single species. Carex (8 species), Galium (6), Salix (6), Cirsium (5), Juncus (5), Rumex (5), and Veronica (5) were the species-richest genera. The large species diversity of the analysed flora reflects the diversity of habitats in terms of trophy, humidity, intensity and type of anthropopressure.

The analysed meadows are characterised by high floristic richness in the relatively small area. Substantially fewer species were found in meadows with a similar area situated in the Foothills, i.e. 111 species in Wojkówka, the Dynowskie Foothills (Ziaja \& WójcIK 2014) and 118 species in Odrzechowa, the Bukowskie Foothills (BrągIEL \& TrąB 2013). The meadow and pasture flora of the entire Dynowskie Foothills comprises 363 species (WoLAŃsKi \& TrąBA 2007).

The analysis of the life forms following the classification proposed by Raunkiaer has shown distinct dominance of hemicryptophytes (54\%) and therophytes (19\%) over other forms. Geophytes and hydrophytes accounted for $12 \%$ and $5 \%$, respectively.

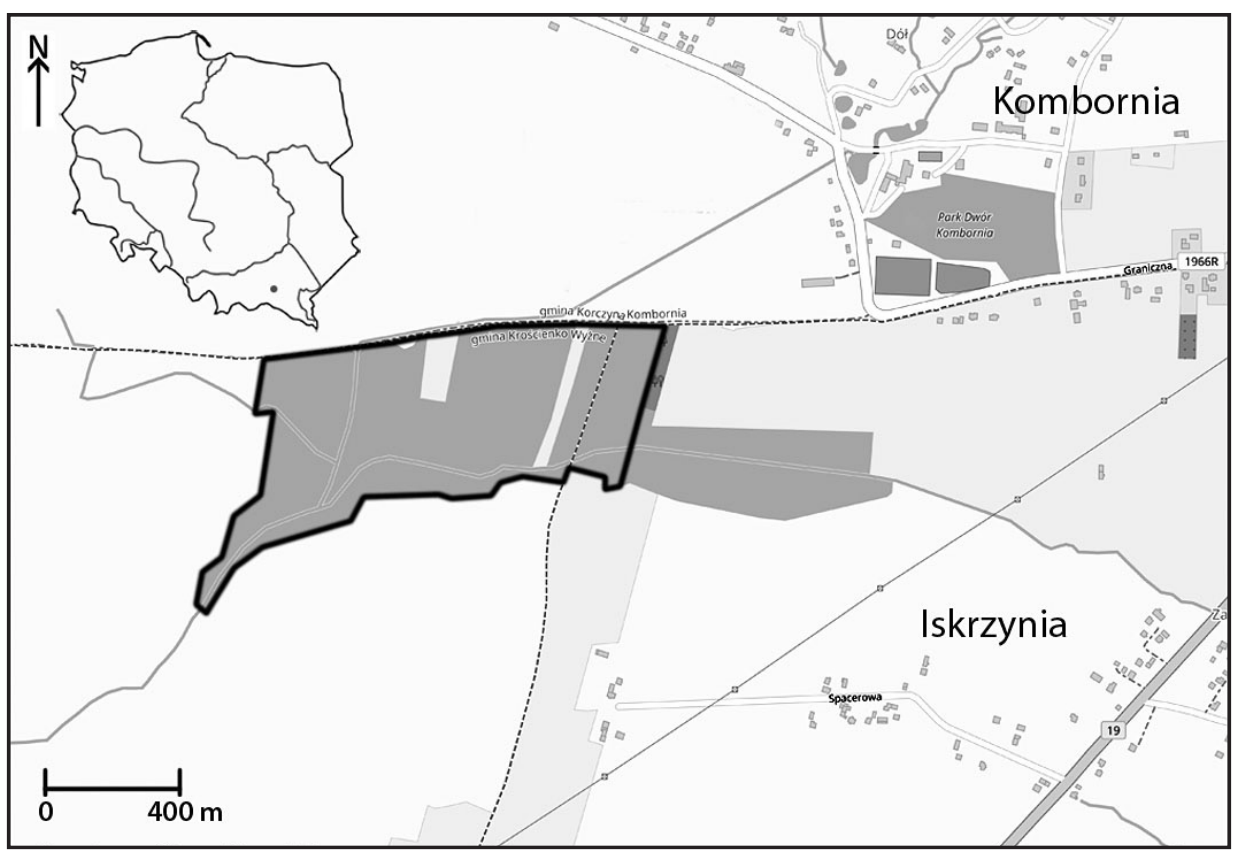

Fig. 1. Study area 
Table 1. Alphabetical list and ecological characterization of vascular plant species (Natura 2000 site Łąki w Komborni)

\begin{tabular}{|c|c|c|c|c|c|c|c|c|}
\hline No & Species & Family & LS & GHs & LF & Phu & \multicolumn{2}{|c|}{ UF } \\
\hline 1 & Achillea millefolium L. s. str. & Asteraceae & $\mathrm{p}$ & Ap & $\mathrm{H}$ & M-A & M & $\mathrm{N}, \mathrm{P}$ \\
\hline 2 & Aegopodium podagraria L. & Apiaceae & $\mathrm{p}$ & Ap & $\mathrm{H}$ & Q-F & M & $\mathrm{N}, \mathrm{P}$ \\
\hline 3 & Agrostis capillaris L. & Poaceae & $\mathrm{p}$ & Ap & $\mathrm{H}$ & $\mathrm{N}-\mathrm{C}$ & - & - \\
\hline 4 & Ajuga reptans L. & Lamiaceae & $\mathrm{p}$ & Ap & $\mathrm{H}$ & Q-F & M & $\mathrm{N}, \mathrm{P}$ \\
\hline 5 & Alchemilla monticola Opiz & Rosaceae & $\mathrm{p}$ & Ap & $\mathrm{H}$ & M-A & M & - \\
\hline 6 & Alisma plantago-aquatica $\mathrm{L}$. & Alismataceae & $\mathrm{p}$ & Ap & Hy & $\mathrm{Ph}$ & - & - \\
\hline 7 & Alliaria petiolata (M. Bieb.) Cavara \& Grande & Brassicaceae & $\mathrm{b}$ & Ap & $\mathrm{H}$ & AR & M & - \\
\hline 8 & Alnus glutinosa (L.) Gaertn. & Betulaceae & $\mathrm{t}$ & Ap & M & AG & M & $P$ \\
\hline 9 & Alopecurus pratensis $\mathrm{L}$. & Poaceae & $\mathrm{p}$ & Ap & $\mathrm{H}$ & M-A & - & - \\
\hline 10 & Anagallis arvensis $\mathrm{L}$. & Primulaceae & $\mathrm{a}$ & Arch & $\mathrm{T}$ & SM & M & - \\
\hline 11 & Angelica sylvestris $\mathrm{L}$. & Apiaceae & $\mathrm{p}$ & Ap & $\mathrm{H}$ & M-A & M & $\mathrm{N}, \mathrm{P}$ \\
\hline 12 & Anthoxanthum odoratum L. s. str. & Poaceae & $\mathrm{p}$ & Ap & $\mathrm{H}$ & M-A & - & - \\
\hline 13 & Apera spica-venti (L.) P. Beauv. & Poaceae & $\mathrm{a}$ & Arch & $\mathrm{T}$ & SM & - & - \\
\hline 14 & Armoracia rusticana P. Gaertn., B. Mey. \& Scherb. & Brassicaceae & $\mathrm{p}$ & Arch & G & AR & M & - \\
\hline 15 & Arrhenatherum elatius (L.) P. Beauv ex J. Presl \& C. Presl & Poaceae & $\mathrm{p}$ & Ap & $\mathrm{H}$ & M-A & - & - \\
\hline 16 & Artemisia vulgaris $\mathrm{L}$. & Asteraceae & $\mathrm{p}$ & Ap & $\mathrm{H}$ & AR & M & - \\
\hline 17 & Atriplex patula L. & Chenopodiaceae & $\mathrm{a}$ & Ap & $\mathrm{T}$ & SM & - & - \\
\hline 18 & Batrachium circinatum (Sibith.) Fr. & Ranunculaceae & $\mathrm{p}$ & Ap & Hy & Po & - & - \\
\hline 19 & Berula erecta (Huds.) Coville & Apiaceae & $\mathrm{p}$ & Ap & Hy & $\mathrm{Ph}$ & - & - \\
\hline 20 & Betonica officinalis L. & Lamiaceae & $\mathrm{p}$ & Ap & $\mathrm{H}$ & M-A & M & $\mathrm{N}, \mathrm{P}$ \\
\hline 21 & Briza media L. & Poaceae & $\mathrm{p}$ & Ap & $\mathrm{H}$ & M-A & - & - \\
\hline 22 & Bromus secalinus L. & Poaceae & $\mathrm{a}$ & Arch & $\mathrm{T}$ & SM & - & - \\
\hline 23 & Bryonia alba L. & Cucurbitaceae & $\mathrm{p}$ & $\mathrm{Kn}$ & $\mathrm{H}$ & - & M & - \\
\hline 24 & Calamagrostis epigejos (L.) Roth & Poaceae & $\mathrm{p}$ & Ap & G & EA & - & - \\
\hline 25 & Caltha palustris L. & Ranunculaceae & $\mathrm{p}$ & Ap & $\mathrm{H}$ & M-A & - & $P$ \\
\hline 26 & Calystegia sepium (L.) R. Br. & Convolvulaceae & $\mathrm{p}$ & Ap & G & AR & - & $\mathrm{N}, \mathrm{P}$ \\
\hline 27 & Campanula patula L. s. str. & Campanulaceae & $\mathrm{b}$ & Ap & $\mathrm{H}$ & M-A & - & $\mathrm{N}, \mathrm{P}$ \\
\hline 28 & Campanula trachelium $\mathrm{L}$. & Campanulaceae & $\mathrm{p}$ & Ap & $\mathrm{H}$ & Q-F & - & $\mathrm{N}, \mathrm{P}$ \\
\hline 29 & Capsella bursa-pastoris (L.) Medik. & Brassicaceae & a & Arch & $\mathrm{T}$ & SM & M & - \\
\hline 30 & Carduus acanthoides L. & Asteraceae & $\mathrm{b}$ & Arch & $\mathrm{H}$ & $\mathrm{AR}$ & - & $\mathrm{N}, \mathrm{P}$ \\
\hline 31 & Carex flava $\mathrm{L}$. & Cyperaceae & $\mathrm{p}$ & Ap & $\mathrm{H}$ & M-A & - & - \\
\hline 32 & Carex gracilis Curtis & Cyperaceae & $\mathrm{p}$ & Ap & G & $\mathrm{Ph}$ & - & - \\
\hline 33 & Carex hirta L. & Cyperaceae & $\mathrm{p}$ & Ap & G & M-A & - & - \\
\hline 34 & Carex nigra Reichard & Cyperaceae & $\mathrm{p}$ & Ap & G & $\mathrm{Ph}$ & - & - \\
\hline 35 & Carex ovalis Gooden. & Cyperaceae & $\mathrm{p}$ & Ap & $\mathrm{H}$ & - & - & - \\
\hline 36 & Carex spicata Huds. & Cyperaceae & $\mathrm{p}$ & Ap & $\mathrm{H}$ & - & - & - \\
\hline 37 & Carex tomentosa $\mathrm{L}$. & Cyperaceae & $\mathrm{p}$ & Ap & G & M-A & - & - \\
\hline 38 & Carex vulpina L. & Cyperaceae & $\mathrm{p}$ & Ap & $\mathrm{H}$ & $\mathrm{Ph}$ & - & - \\
\hline 39 & Centaurea cyanus L. & Asteraceae & a & Arch & $\mathrm{T}$ & SM & M & $\mathrm{N}, \mathrm{P}$ \\
\hline 40 & Centaurea jacea L. & Asteraceae & $\mathrm{p}$ & Ap & $\mathrm{H}$ & M-A & M & $\mathrm{N}, \mathrm{P}$ \\
\hline 41 & Centhaurium erythraea Rafn (PS) & Gentianaceae & $\mathrm{b}$ & Ap & $\mathrm{T}$ & EA & M & $=$ \\
\hline 42 & Cerastium holosteoides Fr. emend. Hyl. & Caryophyllaceae & a & Ap & $\mathrm{C}$ & M-A & - & $\mathrm{N}, \mathrm{P}$ \\
\hline 43 & Chaerophyllum aromaticum $\mathrm{L}$. & Apiaceae & $\mathrm{p}$ & Ap & $\mathrm{H}$ & $\mathrm{AR}$ & - & $\mathrm{N}, \mathrm{P}$ \\
\hline 44 & Chamomilla suaveolens (Pursh) Rydb. & Asteraceae & $\mathrm{a}$ & $\mathrm{Kn}$ & $\mathrm{T}$ & M-A & M & $\mathrm{N}, \mathrm{P}$ \\
\hline 45 & Chenopodium album $\mathrm{L}$. & Chenopodiaceae & a & Ap & $\mathrm{T}$ & SM & M & $\mathrm{P}$ \\
\hline 46 & Chenopodium polyspermum $\mathrm{L}$. & Chenopodiaceae & a & Ap & $\mathrm{T}$ & SM & - & $\mathrm{P}$ \\
\hline 47 & Cirsium arvense (L.) Scop. & Asteraceae & $\mathrm{p}$ & Ap & G & AR & - & $\mathrm{N}, \mathrm{P}$ \\
\hline 48 & Cirsium canum (L.) All. & Asteraceae & $\mathrm{p}$ & Ap & $\mathrm{H}$ & M-A & - & $\mathrm{N}, \mathrm{P}$ \\
\hline 49 & Cirsium oleraceum (L.) Scop. & Asteraceae & $\mathrm{p}$ & Ap & $\mathrm{H}$ & M-A & M & $\mathrm{N}, \mathrm{P}$ \\
\hline 50 & Cirsium palustre (L.) Scop. & Asteraceae & $\mathrm{b}$ & Ap & $\mathrm{H}$ & M-A & - & $\mathrm{N}, \mathrm{P}$ \\
\hline 51 & Cirsium rivulare (Jacq.) All. & Asteraceae & $\mathrm{p}$ & Ap & $\mathrm{H}$ & M-A & - & $\mathrm{N}, \mathrm{P}$ \\
\hline 52 & Convolvulus arvensis $\mathrm{L}$. & Convolvulaceae & $\mathrm{p}$ & Ap & G & AI & - & $\mathrm{N}, \mathrm{P}$ \\
\hline 53 & Conyza canadensis (L.) Cronquist & Asteraceae & $\mathrm{a}$ & $\mathrm{Kn}$ & $\mathrm{T}$ & SM & M & $\mathrm{P}$ \\
\hline 54 & Crataegus monogyna Jacq. & Rosaceae & $\mathrm{s}$ & Ap & $\mathrm{N}$ & R-P & M & $\mathrm{N}, \mathrm{P}$ \\
\hline 55 & Crepis biennis $\mathrm{L}$. & Asteraceae & $\mathrm{b}$ & Ap & $\mathrm{H}$ & M-A & M & $\mathrm{N}, \mathrm{P}$ \\
\hline 56 & Cruciata glabra (L.) Ehrend. & Rubiaceae & $\mathrm{p}$ & Ap & $\mathrm{H}$ & Q-F & - & - \\
\hline
\end{tabular}




\begin{tabular}{|c|c|c|c|c|c|c|c|c|}
\hline No & Species & Family & LS & GHs & $\mathrm{LF}$ & Phu & \multicolumn{2}{|c|}{ UF } \\
\hline 57 & Cucubalus baccifer L. & Caryophyllaceae & $\mathrm{p}$ & Ap & $\mathrm{H}$ & $\mathrm{AR}$ & - & $\mathrm{N}, \mathrm{P}$ \\
\hline 58 & Dactylis glomerata $\mathrm{L}$. & Poaceae & $\mathrm{p}$ & Ap & $\mathrm{H}$ & M-A & - & - \\
\hline 59 & Dactylorhiza majalis (Rchb.) P.F. Hunt \& Summerh. (PS) & Orchidaceae & $\mathrm{p}$ & Ap & G & M-A & - & $\mathrm{N}, \mathrm{P}$ \\
\hline 60 & Daucus carota $\mathrm{L}$. & Apiaceae & $\mathrm{b}$ & Ap & $\mathrm{H}$ & M-A & M & $\mathrm{N}, \mathrm{P}$ \\
\hline 61 & Deschampsia caespitosa (L.) P. Beauv. & Poaceae & $\mathrm{p}$ & Ap & $\mathrm{H}$ & M-A & - & - \\
\hline 62 & Echinochloa crus-galli (L.) P. Beauv. & Poaceae & a & Arch & $\mathrm{T}$ & SM & - & - \\
\hline 63 & Echinocystis lobata (F. Michx.) Torr. \& A. Gray & Cucurbitaceae & $\mathrm{a}$ & $\mathrm{Kn}$ & $\mathrm{T}$ & SP & - & - \\
\hline 64 & Elymus caninus (L.) L. & Poaceae & $\mathrm{p}$ & Ap & $\mathrm{H}$ & AR & M & - \\
\hline 65 & Elymus repens (L.) Gould & Poaceae & $\mathrm{p}$ & Ap & G & $\mathrm{AI}$ & M & - \\
\hline 66 & Epilobium hirsutum L. & Onagraceae & $\mathrm{p}$ & Ap & $\mathrm{H}$ & $\mathrm{Ph}$ & - & $\mathrm{N}, \mathrm{P}$ \\
\hline 67 & Epilobium palustre L. & Onagraceae & $\mathrm{p}$ & Ap & $\mathrm{H}$ & $\mathrm{Ph}$ & - & $\mathrm{N}, \mathrm{P}$ \\
\hline 68 & Equisetum arvense $\mathrm{L}$. & Equisetaceae & $\mathrm{p}$ & Ap & G & AI & M & - \\
\hline 69 & Equisetum fluviatile L. & Equisetaceae & $\mathrm{p}$ & Ap & Hy & $\mathrm{Ph}$ & - & - \\
\hline 70 & Equisetum palustre L. & Equisetaceae & $\mathrm{p}$ & Ap & G & M-A & - & - \\
\hline 71 & Erigeron annuus (L.) Pers. & Asteraceae & $\mathrm{b}$ & $\mathrm{Kn}$ & $\mathrm{H}$ & - & - & $\mathrm{N}, \mathrm{P}$ \\
\hline 72 & Euonymus europaea L. & Celastraceae & s & Ap & $\mathrm{N}$ & Q-F & M & $\mathrm{N}, \mathrm{P}$ \\
\hline 73 & Eupatorium cannabinum $\mathrm{L}$. & Asteraceae & $\mathrm{p}$ & Ap & $\mathrm{H}$ & AR & M & $\mathrm{N}, \mathrm{P}$ \\
\hline 74 & Fallopia convolvulus (L.) Á. Löve & Polygonaceae & $\mathrm{a}$ & Arch & $\mathrm{T}$ & SM & - & $\mathrm{N}, \mathrm{P}$ \\
\hline 75 & Festuca pratensis Huds. & Poaceae & $\mathrm{p}$ & Ap & $\mathrm{H}$ & M-A & - & - \\
\hline 76 & Festuca rubra L. s. str. & Poaceae & $\mathrm{p}$ & Ap & $\mathrm{H}$ & M-A & - & - \\
\hline 77 & Filipendula ulmaria (L.) Maxim. & Rosaceae & $\mathrm{p}$ & Ap & $\mathrm{H}$ & M-A & M & $\mathrm{P}$ \\
\hline 78 & Galeopsis bifida Boenn. & Lamiaceae & $\mathrm{a}$ & Ap & $\mathrm{T}$ & SM & M & $\mathrm{N}, \mathrm{P}$ \\
\hline 79 & Galeopsis speciosa Mill. & Lamiaceae & $\mathrm{a}$ & Ap & $\mathrm{T}$ & SM & M & $\mathrm{N}, \mathrm{P}$ \\
\hline 80 & Galeopsis tetrahit L. & Lamiaceae & $\mathrm{a}$ & Ap & $\mathrm{T}$ & SM & M & $\mathrm{N}, \mathrm{P}$ \\
\hline 81 & Galium aparine L. & Rubiaceae & $\mathrm{a}$ & Ap & $\mathrm{T}$ & $\mathrm{AR}$ & - & $\mathrm{N}, \mathrm{P}$ \\
\hline 82 & Galium boreale $\mathrm{L}$. & Rubiaceae & $\mathrm{p}$ & Ap & $\mathrm{H}$ & M-A & - & $\mathrm{N}, \mathrm{P}$ \\
\hline 83 & Galium mollugo L. s. str. & Rubiaceae & $\mathrm{p}$ & Ap & $\mathrm{H}$ & M-A & - & $\mathrm{N}, \mathrm{P}$ \\
\hline 84 & Galium palustre L. & Rubiaceae & $\mathrm{p}$ & Ap & $\mathrm{H}$ & $\mathrm{Ph}$ & - & $\mathrm{N}, \mathrm{P}$ \\
\hline 85 & Galium spurium L. subsp. spurium & Rubiaceae & a & Arch & $\mathrm{T}$ & - & - & $\mathrm{N}, \mathrm{P}$ \\
\hline 86 & Galium verum L. s. str. & Rubiaceae & $\mathrm{p}$ & Ap & $\mathrm{H}$ & T-G & M & $\mathrm{N}, \mathrm{P}$ \\
\hline 87 & Geranium dissectum $\mathrm{L}$. & Geraniaceae & $\mathrm{a}$ & Arch & $\mathrm{T}$ & SM & - & $\mathrm{N}, \mathrm{P}$ \\
\hline 88 & Geranium palustre L. & Geraniaceae & $\mathrm{p}$ & Ap & $\mathrm{H}$ & M-A & - & $\mathrm{N}, \mathrm{P}$ \\
\hline 89 & Geranium pratense $\mathrm{L}$. & Geraniaceae & $\mathrm{p}$ & Ap & $\mathrm{H}$ & M-A & - & $\mathrm{N}, \mathrm{P}$ \\
\hline 90 & Geum rivale $\mathrm{L}$. & Rosaceae & $\mathrm{p}$ & Ap & $\mathrm{H}$ & M-A & M & $\mathrm{N}, \mathrm{P}$ \\
\hline 91 & Geum urbanum L. & Rosaceae & $\mathrm{p}$ & Ap & $\mathrm{H}$ & $\mathrm{AR}$ & M & - \\
\hline 92 & Gladiolus imbricatus L. (PS) & Iridaceae & $\mathrm{p}$ & Ap & G & M-A & - & $P$ \\
\hline 93 & Glechoma hederacea L. & Lamiaceae & $\mathrm{p}$ & Ap & $\mathrm{H}$ & AR & M & $\mathrm{N}, \mathrm{P}$ \\
\hline 94 & Gnaphalium uliginosum $\mathrm{L}$. & Asteraceae & $\mathrm{a}$ & Ap & $\mathrm{T}$ & $\mathrm{I}-\mathrm{N}$ & - & - \\
\hline 95 & Heracleum sphondylium L. s. str. & Apiaceae & $\mathrm{p}$ & Ap & $\mathrm{H}$ & M-A & M & $\mathrm{N}, \mathrm{P}$ \\
\hline 96 & Hieracium umbellatum $\mathrm{L}$. & Asteraceae & $\mathrm{p}$ & Ap & $\mathrm{H}$ & $\mathrm{N}-\mathrm{C}$ & - & $\mathrm{N}, \mathrm{P}$ \\
\hline 97 & Holcus lanatus L. & Poaceae & $\mathrm{p}$ & Ap & $\mathrm{H}$ & M-A & - & - \\
\hline 98 & Hypericum maculatum Crantz & Hypericaceae & $\mathrm{p}$ & Ap & $\mathrm{H}$ & B-A & M & $P$ \\
\hline 99 & Hypericum perforatum $\mathrm{L}$. & Hypericaceae & $\mathrm{p}$ & Ap & $\mathrm{H}$ & - & M & $\mathrm{P}$ \\
\hline 100 & Hypericum tetrapterum Fr. & Hypericaceae & $\mathrm{p}$ & Ap & $\mathrm{H}$ & M-A & M & $\mathrm{P}$ \\
\hline 101 & Iris pseudacorus L. & Iridaceae & $\mathrm{p}$ & Ap & Hy & $\mathrm{Ph}$ & M & $\mathrm{N}, \mathrm{P}$ \\
\hline 102 & Juglans regia $\mathrm{L}$. & Juglandaceae & $\mathrm{t}$ & $\mathrm{Kn}$ & $\mathrm{N}$ & - & M & $\mathrm{P}$ \\
\hline 103 & Juncus articulatus L. emend. K. Richt. & Juncaceae & $\mathrm{p}$ & Ap & $\mathrm{H}$ & M-A & - & - \\
\hline 104 & Juncus bufonius L. & Juncaceae & $\mathrm{a}$ & Ap & $\mathrm{T}$ & $\mathrm{I}-\mathrm{N}$ & - & - \\
\hline 105 & Juncus conglomeratus L. emend. Leers & Juncaceae & $\mathrm{p}$ & Ap & $\mathrm{H}$ & M-A & - & - \\
\hline 106 & Juncus effusus L. & Juncaceae & $\mathrm{p}$ & Ap & $\mathrm{H}$ & M-A & - & - \\
\hline 107 & Juncus inflexus L. & Juncaceae & $\mathrm{p}$ & Ap & $\mathrm{H}$ & M-A & - & - \\
\hline 108 & Knautia arvensis (L.) J.M. Coult. & Dipsacaceae & $\mathrm{p}$ & Ap & $\mathrm{H}$ & M-A & M & $\mathrm{N}, \mathrm{P}$ \\
\hline 109 & Lamium album $\mathrm{L}$. & Lamiaceae & $\mathrm{p}$ & Arch & $\mathrm{H}$ & AR & M & $\mathrm{N}, \mathrm{P}$ \\
\hline 110 & Lapsana communis L. s. str. & Asteraceae & $\mathrm{b}$ & Ap & $\mathrm{T}$ & AR & - & $\mathrm{N}, \mathrm{P}$ \\
\hline 111 & Lathyrus pratensis $\mathrm{L}$. & Fabaceae & $\mathrm{p}$ & Ap & $\mathrm{H}$ & M-A & - & $\mathrm{N}, \mathrm{P}$ \\
\hline 112 & Leontodon hispidus L. & Asteraceae & $\mathrm{p}$ & Ap & $\mathrm{H}$ & M-A & - & $\mathrm{N}, \mathrm{P}$ \\
\hline 113 & Leucanthemum vulgare Lam. s. str. & Asteraceae & $\mathrm{p}$ & Ap & $\mathrm{H}$ & M-A & - & $\mathrm{N}, \mathrm{P}$ \\
\hline
\end{tabular}




\begin{tabular}{|c|c|c|c|c|c|c|c|c|}
\hline No & Species & Family & LS & GHs & LF & Phu & \multicolumn{2}{|c|}{ UF } \\
\hline 114 & Lolium perenne $\mathrm{L}$. & Poaceae & $\mathrm{p}$ & Ap & $\mathrm{H}$ & M-A & - & - \\
\hline 115 & Lotus corniculatus L. & Fabaceae & $\mathrm{p}$ & Ap & $\mathrm{H}$ & M-A & - & $\mathrm{N}, \mathrm{P}$ \\
\hline 116 & Lotus uliginosus Schkuhr & Fabaceae & $\mathrm{p}$ & Ap & $\mathrm{H}$ & M-A & - & $\mathrm{N}, \mathrm{P}$ \\
\hline 117 & Luzula campestris (L.) DC. & Juncaceae & $\mathrm{p}$ & Ap & $\mathrm{H}$ & $\mathrm{N}-\mathrm{C}$ & - & - \\
\hline 118 & Lychnis flos-cuculi L. & Caryophyllaceae & $\mathrm{p}$ & Ap & $\mathrm{H}$ & M-A & - & $\mathrm{N}, \mathrm{P}$ \\
\hline 119 & Lycopus europaeus L. & Lamiaceae & $\mathrm{p}$ & Ap & Hy & AG & M & $\mathrm{N}, \mathrm{P}$ \\
\hline 120 & Lysimachia nummularia L. & Primulaceae & $\mathrm{p}$ & Ap & $\mathrm{C}$ & M-A & - & $\mathrm{N}, \mathrm{P}$ \\
\hline 121 & Lysimachia vulgaris L. & Primulaceae & $\mathrm{p}$ & Ap & $\mathrm{H}$ & M-A & - & $\mathrm{N}, \mathrm{P}$ \\
\hline 122 & Lythrum salicaria $\mathrm{L}$. & Lythraceae & $\mathrm{p}$ & Ap & $\mathrm{H}$ & M-A & M & $\mathrm{N}, \mathrm{P}$ \\
\hline 123 & Matricaria maritima subsp. inodora (L.) Dostál & Asteraceae & a & Arch & $\mathrm{T}$ & SM & - & $\mathrm{N}, \mathrm{P}$ \\
\hline 124 & Medicago lupulina L. & Fabaceae & $\mathrm{b}$ & Ap & $\mathrm{H}$ & M-A & - & $\mathrm{N}, \mathrm{P}$ \\
\hline 125 & Melandrium album (Mill.) Garcke & Caryophyllaceae & a & Arch & $\mathrm{T}$ & AR & - & $\mathrm{N}, \mathrm{P}$ \\
\hline 126 & Mentha aquatica L. & Lamiaceae & $\mathrm{p}$ & Ap & Hy & $\mathrm{Ph}$ & - & $\mathrm{N}, \mathrm{P}$ \\
\hline 127 & Mentha arvensis L. & Lamiaceae & $\mathrm{p}$ & Ap & G & - & M & $\mathrm{N}, \mathrm{P}$ \\
\hline 128 & Mentha longifolia (L.) L. & Lamiaceae & $\mathrm{p}$ & Ap & $\mathrm{H}$ & M-A & - & $\mathrm{N}, \mathrm{P}$ \\
\hline 129 & Molinia caeruleae (L.) Moench s. str. & Poaceae & $\mathrm{p}$ & Ap & $\mathrm{H}$ & M-A & - & - \\
\hline 130 & Myosotis arvensis (L.) Hill & Boraginaceae & $\mathrm{a}$ & Arch & $\mathrm{T}$ & SM & - & $\mathrm{N}, \mathrm{P}$ \\
\hline 131 & Myosotis palustris (L.) L. emend. Rchb. & Boraginaceae & $\mathrm{p}$ & Ap & $\mathrm{H}$ & M-A & - & $\mathrm{N}, \mathrm{P}$ \\
\hline 132 & Myosoton aquaticum (L.) Moench & Caryophyllaceae & $\mathrm{p}$ & Ap & G & AR & - & $\mathrm{N}, \mathrm{P}$ \\
\hline 133 & Ononis arvensis L. & Fabaceae & $\mathrm{p}$ & Ap & $\mathrm{H}$ & M-A & - & $\mathrm{P}$ \\
\hline 134 & Oxalis fontana Bunge & Oxalidaceae & $\mathrm{p}$ & $\mathrm{Kn}$ & G & SM & - & $\mathrm{P}$ \\
\hline 135 & Padus avium Mill. & Rosaceae & $\mathrm{t}$ & Ap & $\mathrm{M}$ & Q-F & M & $\mathrm{N}, \mathrm{P}$ \\
\hline 136 & Padus serotina (Ehrh.) Borkh. & Rosaceae & $\mathrm{t}$ & $\mathrm{Kn}$ & $\mathrm{N}$ & - & - & $\mathrm{N}, \mathrm{P}$ \\
\hline 137 & Papaver argemone $\mathrm{L}$. & Papaveraceae & $\mathrm{a}$ & Arch & $\mathrm{T}$ & SM & M & $\mathrm{P}$ \\
\hline 138 & Papaver rhoeas L. & Papaveraceae & $\mathrm{a}$ & Arch & $\mathrm{T}$ & SM & M & $\mathrm{P}$ \\
\hline 139 & Phalaris arundinacea $\mathrm{L}$. & Poaceae & $\mathrm{p}$ & Ap & G & $\mathrm{Ph}$ & - & - \\
\hline 140 & Phleum pratense $\mathrm{L}$. & Poaceae & $\mathrm{p}$ & Ap & $\mathrm{H}$ & M-A & - & - \\
\hline 141 & Phragmites australis (Cav.) Trin. ex Steud. & Poaceae & $\mathrm{p}$ & Ap & G & $\mathrm{Ph}$ & - & - \\
\hline 142 & Pimpinella major (L.) Huds. & Apiaceae & $\mathrm{p}$ & Ap & $\mathrm{H}$ & M-A & M & $\mathrm{N}, \mathrm{P}$ \\
\hline 143 & Plantago intermedia Gilib. & Plantaginaceae & $\mathrm{p}$ & Ap & $\mathrm{T}$ & $\mathrm{I}-\mathrm{N}$ & - & $\mathrm{P}$ \\
\hline 144 & Plantago lanceolata $\mathrm{L}$. & Plantaginaceae & $\mathrm{p}$ & Ap & $\mathrm{H}$ & M-A & M & $\mathrm{P}$ \\
\hline 146 & Plantago major L. s. str. & Plantaginaceae & $\mathrm{p}$ & Ap & $\mathrm{H}$ & M-A & M & $\mathrm{P}$ \\
\hline 146 & Platanthera bifolia (L.) Rich. (PS) & Orchidaceae & $\mathrm{p}$ & Ap & G & - & - & $\mathrm{P}$ \\
\hline 147 & Poa annua $\mathrm{L}$. & Poaceae & a & Ap & $\mathrm{H}$ & M-A & - & - \\
\hline 148 & Poa pratensis L. s. str. & Poaceae & $\mathrm{p}$ & Ap & $\mathrm{H}$ & M-A & - & - \\
\hline 149 & Poa trivialis $\mathrm{L}$. & Poaceae & $\mathrm{p}$ & Ap & $\mathrm{H}$ & M-A & - & - \\
\hline 150 & Polygonum amphibium L. & Polygonaceae & $\mathrm{p}$ & Ap & G & Po & - & $\mathrm{N}, \mathrm{P}$ \\
\hline 151 & Polygonum aviculare $\mathrm{L}$. & Polygonaceae & a & Ap & $\mathrm{T}$ & SM & M & $\mathrm{N}, \mathrm{P}$ \\
\hline 152 & Polygonum lapathifolium L. subsp. lapathifolium & Polygonaceae & a & Ap & $\mathrm{T}$ & BT & - & $\mathrm{N}, \mathrm{P}$ \\
\hline 153 & Polygonum persicaria $\mathrm{L}$. & Polygonaceae & $\mathrm{a}$ & Ap & $\mathrm{T}$ & SM & - & $\mathrm{N}, \mathrm{P}$ \\
\hline 154 & Potentilla anserina $\mathrm{L}$. & Rosaceae & $\mathrm{p}$ & Ap & $\mathrm{H}$ & M-A & M & $\mathrm{P}$ \\
\hline 155 & Potentilla erecta (L.) Raeusch. & Rosaceae & $\mathrm{p}$ & Ap & $\mathrm{H}$ & $\mathrm{N}-\mathrm{C}$ & M & $\mathrm{P}$ \\
\hline 156 & Primula elatior (L.) Hill (PS) & Primulaceae & $\mathrm{p}$ & Ap & $\mathrm{H}$ & Q-F & M & $\mathrm{N}, \mathrm{P}$ \\
\hline 157 & Prunella vulgaris $\mathrm{L}$. & Lamiaceae & $\mathrm{p}$ & Ap & $\mathrm{H}$ & M-A & M & $\mathrm{N}, \mathrm{P}$ \\
\hline 158 & Quercus robur $\mathrm{L}$. & Fagaceae & $\mathrm{t}$ & Ap & M & Q-F & M & $\mathrm{P}$ \\
\hline 159 & Ranunculus acris L. s. str. & Ranunculaceae & $\mathrm{p}$ & Ap & $\mathrm{H}$ & M-A & - & $\mathrm{P}$ \\
\hline 160 & Ranunculus auricomus L. s. 1. & Ranunculaceae & $\mathrm{p}$ & Ap & $\mathrm{H}$ & Q-F & - & $\mathrm{P}$ \\
\hline 161 & Ranunculus flammula L. & Ranunculaceae & $\mathrm{p}$ & Ap & $\mathrm{H}$ & $\mathrm{Ph}$ & - & $\mathrm{P}$ \\
\hline 162 & Ranunculus repens $\mathrm{L}$. & Ranunculaceae & $\mathrm{p}$ & Ap & $\mathrm{H}$ & M-A & - & $\mathrm{P}$ \\
\hline 163 & Rhamnus cathartica $\mathrm{L}$. & Rhamnaceae & s & Ap & $\mathrm{N}$ & R-P & M & $\mathrm{N}, \mathrm{P}$ \\
\hline 164 & Rhus typhina L. & Anacardiacaceae & s & $\mathrm{Kn}$ & $\mathrm{N}$ & - & - & - \\
\hline 165 & Rorippa sylvestris (L.) Besser & Brassicaceae & $\mathrm{p}$ & Ap & G & M-A & - & $\mathrm{N}, \mathrm{P}$ \\
\hline 166 & Rosa canina $\mathrm{L}$. & Rosaceae & $\mathrm{s}$ & Ap & $\mathrm{N}$ & R-P & M & $\mathrm{P}$ \\
\hline 167 & Rubus caesius L. & Rosaceae & $\mathrm{s}$ & Ap & $\mathrm{N}$ & R-P & - & $\mathrm{N}, \mathrm{P}$ \\
\hline 168 & Rumex acetosa $\mathrm{L}$. & Polygonaceae & $\mathrm{p}$ & Ap & $\mathrm{H}$ & M-A & M & $\mathrm{P}$ \\
\hline 169 & Rumex confertus Willd. & Polygonaceae & $\mathrm{p}$ & $\mathrm{Kn}$ & $\mathrm{H}$ & - & - & $\mathrm{P}$ \\
\hline 170 & Rumex crispus L. & Polygonaceae & $\mathrm{p}$ & Ap & $\mathrm{H}$ & M-A & - & $\mathrm{P}$ \\
\hline
\end{tabular}




\begin{tabular}{|c|c|c|c|c|c|c|c|c|}
\hline No & Species & Family & LS & GHs & LF & Phu & \multicolumn{2}{|c|}{ UF } \\
\hline 171 & Rumex hydrolapathum Huds. & Polygonaceae & $\mathrm{p}$ & Ap & Hy & $\mathrm{Ph}$ & - & $\mathrm{P}$ \\
\hline 172 & Rumex obtusifolius L. & Polygonaceae & $\mathrm{p}$ & Ap & $\mathrm{H}$ & AR & M & $\mathrm{P}$ \\
\hline 173 & Salix alba $\mathrm{L}$. & Salicaceae & $\mathrm{t}$ & Ap & M & SP & M & $\mathrm{N}, \mathrm{P}$ \\
\hline 174 & Salix caprea L. & Salicaceae & s & Ap & $\mathrm{N}$ & EA & - & $\mathrm{N}, \mathrm{P}$ \\
\hline 175 & Salix cinerea $\mathrm{L}$. & Salicaceae & s & Ap & $\mathrm{N}$ & AG & - & $\mathrm{N}, \mathrm{P}$ \\
\hline 176 & Salix fragilis $\mathrm{L}$. & Salicaceae & $\mathrm{t}$ & Ap & M & SP & - & $\mathrm{N}, \mathrm{P}$ \\
\hline 177 & Salix purpurea L. & Salicaceae & s & Ap & $\mathrm{N}$ & SP & M & $\mathrm{N}, \mathrm{P}$ \\
\hline 178 & Salix triandra $\mathrm{L}$. & Salicaceae & s & Ap & $\mathrm{N}$ & SP & - & $\mathrm{N}, \mathrm{P}$ \\
\hline 179 & Sambucus nigra L. & Caprifoliaceae & $\mathrm{s}$ & Ap & $\mathrm{N}$ & EA & M & $\mathrm{P}$ \\
\hline 180 & Sanguisorba officinalis L. & Rosaceae & $\mathrm{p}$ & Ap & $\mathrm{H}$ & $\mathrm{M}-\mathrm{A}$ & M & $\mathrm{N}, \mathrm{P}$ \\
\hline 181 & Scirpus sylvaticus L. & Cyperaceae & $\mathrm{p}$ & Ap & G & $\mathrm{M}-\mathrm{A}$ & - & - \\
\hline 182 & Scrophularia nodosa L. & Scrophulariaceae & $\mathrm{p}$ & Ap & $\mathrm{H}$ & Q-F & M & $\mathrm{N}, \mathrm{P}$ \\
\hline 183 & Scuttelaria galericulata L. & Lamiaceae & $\mathrm{p}$ & Ap & $\mathrm{H}$ & $\mathrm{Ph}$ & M & $\mathrm{N}, \mathrm{P}$ \\
\hline 184 & Selinum carvifolia (L.) L. & Apiaceae & $\mathrm{p}$ & Ap & $\mathrm{H}$ & M-A & - & $\mathrm{N}, \mathrm{P}$ \\
\hline 185 & Senecio fluviatilis Wallr. & Asteraceae & $\mathrm{p}$ & Ap & $\mathrm{H}$ & SP & - & $\mathrm{N}, \mathrm{P}$ \\
\hline 186 & Serratula tinctoria L. & Asteraceae & $\mathrm{p}$ & Ap & G & M-A & M & $\mathrm{N}, \mathrm{P}$ \\
\hline 187 & Setaria viridis (L.) P. Beauv. & Poaceae & $\mathrm{a}$ & Arch & $\mathrm{T}$ & SM & - & - \\
\hline 188 & Sinapis arvensis L. & Brassicaceae & $\mathrm{a}$ & Arch & $\mathrm{T}$ & SM & M & $\mathrm{N}, \mathrm{P}$ \\
\hline 189 & Solidago gigantea Aiton & Asteraceae & $\mathrm{p}$ & $\mathrm{Kn}$ & G & $\mathrm{AR}$ & M & $\mathrm{N}, \mathrm{P}$ \\
\hline 190 & Solidago virgaurea $\mathrm{L}$. & Asteraceae & $\mathrm{p}$ & Ap & $\mathrm{H}$ & - & M & $\mathrm{N}, \mathrm{P}$ \\
\hline 191 & Sonchus asper (L.) Hill & Asteraceae & a & Arch & $\mathrm{T}$ & SM & - & $\mathrm{N}, \mathrm{P}$ \\
\hline 192 & Sonchus oleraceus L. & Asteraceae & $\mathrm{p}$ & Arch & $\mathrm{T}$ & SM & - & $\mathrm{N}, \mathrm{P}$ \\
\hline 193 & Sparganium erectum L. emend. Rchb. s. str. & Sparganiaceae & $\mathrm{p}$ & Ap & Hy & $\mathrm{Ph}$ & - & - \\
\hline 194 & Stachys palustris $\mathrm{L}$. & Lamiaceae & $\mathrm{p}$ & Ap & G & M-A & - & $\mathrm{N}, \mathrm{P}$ \\
\hline 195 & Stellaria graminea L. & Cayrophyllaceae & $\mathrm{p}$ & Ap & $\mathrm{H}$ & M-A & - & $\mathrm{N}, \mathrm{P}$ \\
\hline 196 & Succisa pratensis Moench & Dipsacaceae & $\mathrm{p}$ & Ap & $\mathrm{H}$ & M-A & - & $\mathrm{N}, \mathrm{P}$ \\
\hline 197 & Symphytum officinale L. & Boraginaceae & $\mathrm{p}$ & Ap & $\mathrm{H}$ & M-A & M & $\mathrm{N}, \mathrm{P}$ \\
\hline 198 & Tanacetum vulgare $\mathrm{L}$. & Asteraceae & $\mathrm{p}$ & Ap & $\mathrm{H}$ & AR & M & $\mathrm{P}$ \\
\hline 199 & Thalictrum lucidum L. & Ranunculaceae & $\mathrm{p}$ & Ap & $\mathrm{H}$ & M-A & - & $\mathrm{P}$ \\
\hline 200 & Tragopogon orientalis $\mathrm{L}$. & Asteraceae & $\mathrm{b}$ & Ap & $\mathrm{H}$ & M-A & - & $\mathrm{N}, \mathrm{P}$ \\
\hline 201 & Trifolium dubium Sibth. & Fabaceae & $\mathrm{a}$ & Ap & $\mathrm{T}$ & $\mathrm{M}-\mathrm{A}$ & - & $\mathrm{N}, \mathrm{P}$ \\
\hline 202 & Trifolium hybridum $\mathrm{L}$. & Fabaceae & $\mathrm{p}$ & Ap & $\mathrm{H}$ & M-A & - & $\mathrm{N}, \mathrm{P}$ \\
\hline 203 & Trifolium pratense $\mathrm{L}$. & Fabaceae & $\mathrm{p}$ & Ap & $\mathrm{H}$ & M-A & M & $\mathrm{N}, \mathrm{P}$ \\
\hline 204 & Trifolium repens L. & Fabaceae & $\mathrm{p}$ & Ap & $\mathrm{H}$ & M-A & - & $\mathrm{N}, \mathrm{P}$ \\
\hline 205 & Trisetum flavescens (L.) P. Beauv. & Poaceae & $\mathrm{p}$ & Ap & $\mathrm{H}$ & M-A & - & - \\
\hline 206 & Trollius europaeus L. s. str. (PS) & Ranunculaceae & $\mathrm{p}$ & Ap & $\mathrm{H}$ & M-A & M & $\mathrm{N}, \mathrm{P}$ \\
\hline 207 & Tussilago farfara $\mathrm{L}$. & Asteraceae & $\mathrm{p}$ & Ap & G & AI & M & $\mathrm{N}, \mathrm{P}$ \\
\hline 208 & Typha latifolia L. & Typhaceae & $\mathrm{p}$ & Ap & Hy & $\mathrm{Ph}$ & M & - \\
\hline 209 & Urtica dioica $\mathrm{L}$. & Urticaceae & $\mathrm{p}$ & Ap & $\mathrm{H}$ & AR & M & - \\
\hline 210 & Valeriana officinalis L. & Valerianaceae & $\mathrm{p}$ & Ap & $\mathrm{H}$ & M-A & M & $\mathrm{N}, \mathrm{P}$ \\
\hline 211 & Veronica arvensis L. & Scrophulariaceae & $\mathrm{a}$ & Arch & $\mathrm{T}$ & SM & - & $\mathrm{N}, \mathrm{P}$ \\
\hline 212 & Veronica beccabunga $\mathrm{L}$. & Scrophulariaceae & $\mathrm{p}$ & Ap & $\mathrm{C}$ & $\mathrm{Ph}$ & - & $\mathrm{N}, \mathrm{P}$ \\
\hline 213 & Veronica chamaedrys L. s. str. & Scrophulariaceae & $\mathrm{p}$ & Ap & $\mathrm{C}$ & M-A & - & $\mathrm{N}, \mathrm{P}$ \\
\hline 214 & Veronica longifolia L. & Scrophulariaceae & $\mathrm{p}$ & Ap & $\mathrm{H}$ & M-A & - & $\mathrm{N}, \mathrm{P}$ \\
\hline 215 & Veronica persica PoIR. & Scrophulariaceae & $\mathrm{a}$ & $\mathrm{Kn}$ & $\mathrm{T}$ & SM & - & $\mathrm{N}, \mathrm{P}$ \\
\hline 216 & Viburnum opulus L. & Caprifoliaceae & $\mathrm{s}$ & Ap & $\mathrm{N}$ & Q-F & M & $\mathrm{P}$ \\
\hline 217 & Vicia cracca L. & Fabaceae & $\mathrm{p}$ & Ap & $\mathrm{H}$ & M-A & - & $\mathrm{N}, \mathrm{P}$ \\
\hline 218 & Vicia hirsuta (L.) Gray & Fabaceae & $\mathrm{a}$ & Arch & $\mathrm{T}$ & SM & - & $\mathrm{N}, \mathrm{P}$ \\
\hline 219 & Viola arvensis Murray & Violaceae & $\mathrm{a}$ & Arch & $\mathrm{T}$ & SM & - & $\mathrm{N}, \mathrm{P}$ \\
\hline
\end{tabular}

Explanation: LS (Life span): a - annual, b - biennial, p - perennial, s - shrubs, t - trees; GHs (geographical-historical status): Ap - apophytes, Arch - archaeophytes, Kn - kenophytes; LF (Life forms): M - megaphanerophytes, N - nanophanerophytes, C - chamaephytes, H - hemicryptophytes, G - geophytes, T - terophytes; Hy - hydrophytes, Phu (Phytosociological unit): AG - Alnetea glutinosae, AI - Agropyretea intermedio-repentis, AR - Artemisietea vulgaris, B-A - Betulo-Adenostyletea, BT - Bidentetea tripartiti, EA - Epilobietea angustifolii, I-N - Isoëto-Nanojuncetea, M-A - Molinio-Arrhenatheretea, N-C - Nardo-Callunetea, Ph - Phragmitetea, Po - Potametea, Q-F - Querco-Fagetea, R-P - Rhamno-Prunetea, SM - Stellarietea mediae, SP - Salicetea purpurea, T-G - Trifolio-Geranietea sanguinei, UF (Usage form): M - medicinale species, $\mathrm{N}$ - nectariferous, $\mathrm{P}$ - polleniferous, PS (protected species). 
Phanerophytes made up $8 \%$, with dominance of nanophanerophytes $(6 \%)$ over megaphanerophytes (2\%) (Table 2).

The most numerous group were perennials (150 species, 69\%) typical for grassland ecosystems. Annual and biennial species were represented by 50 species $(23 \%)$. The total number of shrubs and trees were 19 species 8\%) (Table 2). Their occurrence in meadow communities indicates abandonment of land management and initiation of the succession process (FALIŃSKA 1991, Bator 2005, Barabasz-Krasny 2011, TrąBA \& WOLAŃSKI 2012). To maintain stability, meadow communities require human activity (grazing, mowing). Abandonment of these measures results in disturbance in the biological rhythm of meadow plants and encroachment of tree and shrub seedlings. A similar phenomenon was observed in the Dynowskie Foothills (WolańsKi \& Trąa 2007), Przemyskie Foothills (BARABASZ-Krasny 2011), and Wielickie Foothills (BATOR 2005). The early stage of succession can initially contribute to an increase in species diversity followed by a decline in the number of species, as reported by many researchers (FALIŃSKA 1991, BARABASZ-KRASNY 2011, SienKieWicz-PAdereWSKA et al. 2012).

The analysis of the geographical and historical groups revealed considerable dominance of apophytes (183 species, 84\%) over anthropophytes (36 species, 16\%) (Table 2). Among alien species, archaeophytes were a majority (24 species, $11 \%$ ); these usually were species migrating from nearby cereal crop (Apera spica-venti, Matricaria maritima subsp. inodora, Papaver rhoeas, Centaurea cyanus) and root crop (Echinochloa crus-galli, Setaria viridis, Sonchus oleraceus) fields. Kenophytes were represented by 12 species $(5 \%)$; this group comprised commonly occurring invasive species, which posed no threat in the study area, i.e. Echinocystis lobata, Erigeron annuus, Conyza canadensis, Juglans regia, Solidago gigantea, Padus serotina, Rhus typhina and Rumex confertus. Discontinuation of management of meadow communities leads to transformations of the habitat, which in turn triggers colonisation by species from other habitats and enhances migration of alien species (FALIŃSKA 1991, BARABASZ-KRASNY 2011).

In terms of the phytosociological classification, 206 (94\%) of the total species found in the study area are typical species or distinctive syntaxonomic units representing 16 classes. The largest group comprised species from the class Molinio-Arrhenatheretea (92 species, 42\%) (Table 2). Synanthropic species from the classes Stellarietea mediae and Artemisietea vulgaris accounted for $23 \%$ of the total flora. Rush vegetation from the class Phragmitetea was represented by 19 species (9\%), which indicates fertile, flooded, and waterlogged habitats. Due to the lack of management, there were empty spaces in the sward, which were colonised by vegetation from areas ad-
Table 2. Floristic diversification Natura 2000 site "Łąki w Komborni"

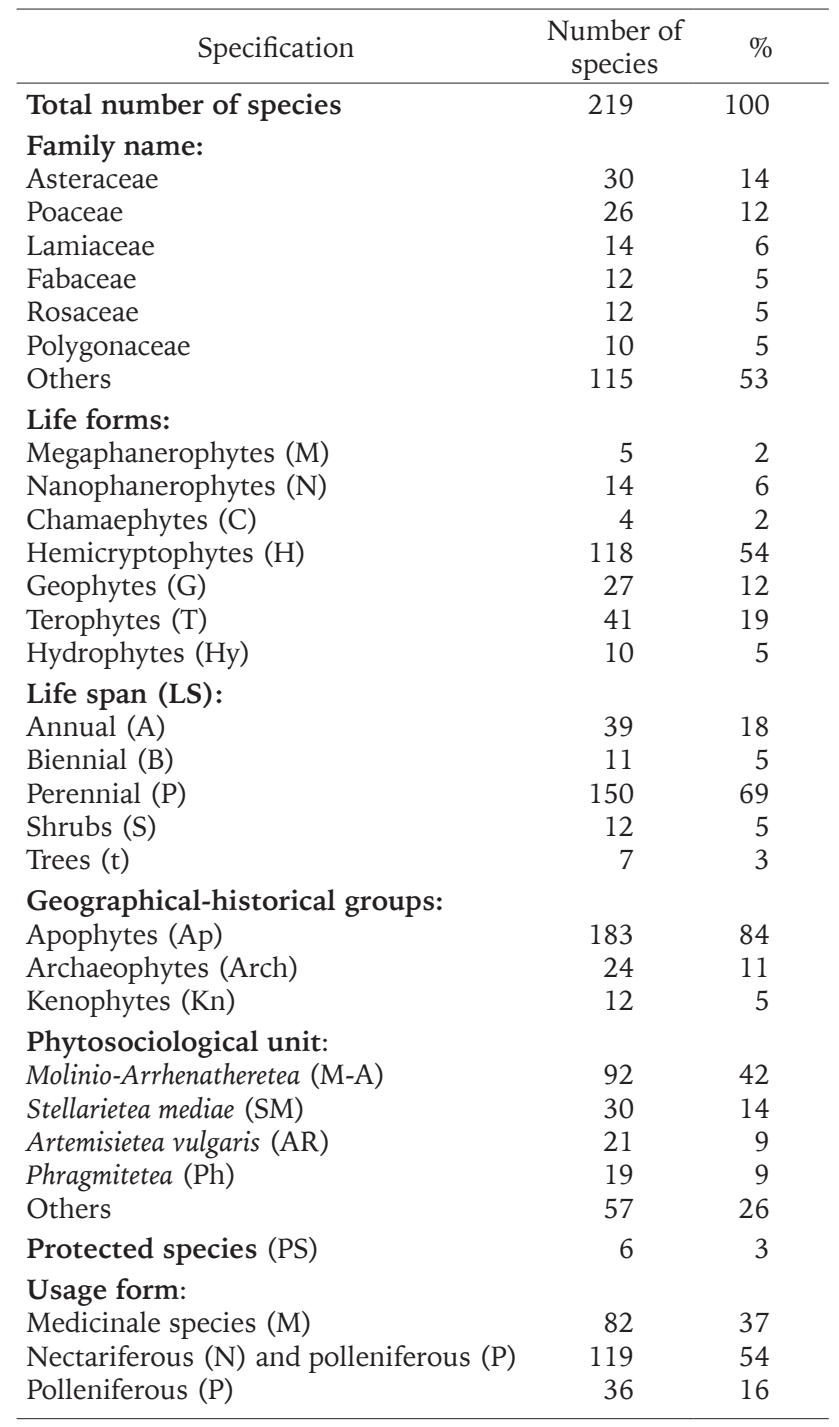

jacent to meadows; hence, the high share of synanthropic species, which has been reported from the Przemyskie Foothills (BARABASZ-Krasny 2011) and Wielickie Foothills (BATOR 2005).

Eighty-two medicinal taxa $(37 \%)$ were found in the analysed meadows, 155 taxa (70\%) represented melliferous (nectariferous and polleniferous) plants providing reward to insects. The high vascular species diversity in the analysed area ensures continuity of the food chain from early spring to late summer.

Particularly noteworthy are protected species, with two under strict protection, i.e. Gladiolus imbricatus and Trollius europaeus, and four under partial protection, i.e. Centhaurium erythraea, Dactylorhiza majalis, Platanthera bifolia and Primula elatior. The presence of rare and protected plants enhances the nature and landscape values of meadow communities, which has been highlighted by many authors (KĄCKI \& MichALSKa-Hejduk 2010, KotańsKa et al. 2015, Ziaja \& WójCIK 2016a, b). 


\section{REFERENCES}

BABCZYŃSKA-SENDEK B. (2009): Significance of protection of the meadow and grassland communities for maintenance the floristic diversity in the area of the south-eastern Silesiana Upland (Poland). Biodiversity: Research and Conservation 13: 4960.

Barabasz-Krasny B. (2011): Zróżnicowanie roślinności i sukcesja wtórna na odłogach wielkopowierzchniowych Pogórza Przemyskiego. Instytut Botaniki im. W. Szafera, Polska Akademia Nauk, Kraków.

BATOR K. (2005): Stan obecny i przemiany zbiorowisk łąkowych okolic Mogilan (Pogórze Wielickie) w okresie 40 lat. Fragmenta Floristica et Geobotanica Polonica, Supplement 7: 1-97.

Brągiel P., Trąba C. (2013): Flora łąk Zakładu Doświadczalnego Instytutu Zootechniki w Odrzechowej objętych programem rolnośrodowiskowym. Woda-Środowisko-Obszary Wiejskie 13, 1(41): 15-30.

Denisow B., Wrzesień M. (2007): The anthropogenic refuge areas for bee flora in agricultural landscape. Acta Agrobotanica 60: 147-157.

ElLENBERG H. (1988): Vegetation ecology of Central Europe. Cambridge University Press, CambridgeNew York-New Rochelle-Melbourne-Sydney.

European Red List of Habitats. (2016). Part 2. Terrestrial and freshwater habitats. http://ec.europa.eu/ environment/nature/knowledge/redlist_en.htm

FALIŃSKA K. (1991): Sukcesja jako efekt procesów demograficznych roślin. In: J.B. Faliński (ed.). Dynamika roślinności i populacji roślinnych. Phytocoenosis 3 (N.S.) Seminarium Geobotanicum 1: 43-67.

KĄCKI Z. (2012): Variability and long-term changes in the species composition of Molinia meadows in Poland: a case study using a large data set from the Polish Vegetation Database. Acta Botanica Silesiaca, Monographiae 7: 1-144.

KącKi Z., MichalsKa-HejDuk D. (2010): Assessment of biodiversity of Molinia meadows in Kampinoski National Parks based on biocoenotic indicators. Polish Journal of Environmental Study 19(2): 351-362.

KondRACKI J. (2012): Geografia regionalna Polski. Wydawnictwo Naukowe PWN, Warszawa.

KotaŃSKA M. (1993): Response of wet meadows of the Calthion alliance to variations of weather and management practices - a thirteen-year study of permanent plots. Studia Naturae 40: 1-47.

Kotańska M., Towpasz K., Wójcik T., MitKa J. (2015): Łąki w krajobrazie rolniczym Płaskowyżu Proszowickiego (Wyżyna Małopolska). Ekologia i Technika 23(4): 182-192.
KRYSZAK J., KRYSZAK A. (2007): Użytkowanie a walory przyrodnicze zbiorowisk łąkowych. Fragmenta Agronomica 24(3): 258-267.

KulIK M. (2014): Changes of biodiversity and species composition of Molinia meadow depending on use method. Polish Journal of Environmental Study 23(3): 773-782.

Lindborg R., Bengtsson J., Berg A., Cousins S.A.O., Eriksson O., Gustafsson T., Hausund K.P., Lenoir L., Pihlgren A., Sjödin E., Stenseke M. (2008): A landscape perspective on conservation of semi-natural grasslands. Agriculture, Ecosystems and Environment 125: 213-222.

LIPIŃski M. (1958): Pożytki pszczele - zapylanie i miododajność roślin. Państwowe Wydawnictwo Rolnicze i Leśne, Warszawa.

Matuszkiewicz W. (2005): Przewodnik do oznaczania zbiorowisk roślinnych Polski. Wydawnictwo Naukowe PWN, Warszawa.

Michalska-Hejduk D., Kopeć D. (2012): Dynamics of semi-natural vegetation with a focus on Molinion meadows after 50 years of strict protection. Polish Journal of Environmental Study 21(6): 17311741.

Mirek Z., Piękoś-Mirkowa H., Zając A., Zając M. (2002): Flowering plants and pteridophytes of Poland. A checklist. Vol. 1. Biodiversity of Poland. - Krytyczna lista roślin naczyniowych Polski. T. 1. Różnorodność biologiczna Polski. W. Szafer Institute of Botany, Polish Academy of Sciences, Kraków.

NowaK T., WęGrZynek B., ToKarsKa-GuziK B. (2015): Assets and threats to Molinia meadows (Molinion caeruleae alliance) on chosen Natura 2000 areas in the eastern part of the Silesian Upland. Acta Scientiarum Polonorum, Agricultura 14(4): 49-61.

Poschlod P., Bakker J.P., Kahmen S. (2005): Changing land use and its impact on biodiversity. Basic and Applied Ecology 6(2): 93-98.

PZO Łąki w Komborni PLH 180042. http://rzeszow. rdos.gov.pl/laki-w-komborni-plh180042.

RutKowski L. (2004): Klucz do oznaczania roślin naczyniowych Polski niżowej. Wydawnictwo Naukowe PWN, Warszawa.

SienKiewicz-PaderewsKa D., BorawsKa-Jarmułowicz B., Mastalerczuk G., Chodkiewicz A., Stypiński P. (2012): Wpływ zaprzestania koszenia na roślinność łąki trzęślicowej (Molinietum caerulae). WodaŚrodowisko-Obszary Wiejskie 12(1): 167-179.

Spychalski W., Kryszak J., KrYszaK A. (2011): Gleby łąk dolinowych i śródpolnych a ich różnorodność florystyczna. Roczniki Gleboznawcze 62(2): 376386.

TOKARSKA-GuZIK B. (2005): The establishment and spreading of alien plant species (kenophytes) in the flora of Poland. Wydawnictwo Uniwersytetu Śląskiego, Katowice. 
TrĄBA C., Wolański P. (2012): Zróżnicowanie florystyczne zbiorowisk łąkowych ze związków Molinion, Cnidion dubii i Filipendulion w Polsce - zagrożenia i ochrona. Inżynieria Ekologiczna 29: 224-235.

Wójcik T., JANICKA M. (2016): Current state and changes in Molinion meadows from Kostrze environs in Kraków. Ecological Questions 23: 15-27.

Wolański P., TrąBA C. (2007): Flora łąk i pastwisk Pogórza Dynowskiego. Woda-Środowisko-Obszary Wiejskie 7, 26(21): 195-204.

Wrzesień M., Denisow B. (2006a): The share of nectariferous and polleniferous taxons in chosen patches of thermophilous grasslands of the Lublin Upland. Acta Agrobotanica 59(1): 213-221.

Wrzesień M., Denisow B. (2006b): The usable taxons in spontaneous flora of railway areas of the central-eastern part of Poland. Acta Agrobotanica 59(2): 95-108.

ZAJAcC A. (1979): Pochodzenie archeofitów występujących w Polsce. Zeszyty Naukowe Uniwersytetu Jagiellońskiego, Rozprawy Habilitacyjne 29: $1-213$.

ZAŁUSKI T. (2007): Zagrożenia i ochrona zespołów trawiastych. In: L. Frey (ed.). Księga polskich traw. Instytut Botaniki im. W. Szafera, Polska Akademia Nauk, Kraków: 283-316.
ZAŁUSKI T. (2011): Vegetation transformations of Kujawy-Pomerania region in the last twenty years period. Acta Universitatis Lodziensis, Folia Biologica et Oecologica 7: 75-98.

ZARZYCKI J., KORZENIAK J. (2013): Łąki w polskich Karpatach - stan aktualny, zmiany i możliwości ich zachowania. Roczniki Bieszczadzkie 21: 18-34.

Zarzycki K., TrzcińSKa-TACIK H., RóżAŃSKi W., SzelĄG Z., WoŁeK J., KorzeniaK U. (2002): Ecological indicator of vascular plants of Poland. Vol. 2. Biodiversity of Poland. - Ekologiczne liczby wskaźnikowe roślin naczyniowych Polski. T. 2. Różnorodność biologiczna Polski. W. Szafer Institute of Botany, Polish Academy of Sciences, Kraków.

ZiAjA M., Wójcik T. (2014): Thermophilic plant communities in Natura 2000 site "Łąki nad Wojkówką" PLH 180051 - Podkarpacie Province. Annales Sectio C 69(1): 59-78.

ZiAjA M., WóJcik T. (2016a): Occurrence of the globeflower Trollius europaeus L. in "Łąki w Komborni" Natura 2000 site (Podkarpackie Province, SE Poland). Ecological Questions 3: 61-69.

ZiajA M., WójCiK T. (2016b): Występowanie Gladiolus imbricatus L. (Iridaceae) w zbiorowiskach łąkowych w obszarze Natura 2000 „Łąki w Komborni” PLH180042 (SE Poland). Fragmenta Floristica et Geobotanica Polonica 23(2): 219-230. 